\title{
Power Engineering Construction Technology and Management
}

\author{
Jianying Li \\ Yunnan Energy Vocational and Technical College, Qujing, Yunnan, 655001
}

Keywords: power engineering construction; management and improvement

\begin{abstract}
In the power field, construction technology and construction management work play a decisive role in the smooth development and good operation of power engineering. Among them, construction technology is the foundation of power engineering development, and construction management is the guarantee to improve the efficiency of power engineering operation. The power engineering unit should strengthen the technical management of power engineering, improve the professional quality of engineering personnel, and pay attention to the information construction and pre-design work of power engineering, thus contributing to the development of the power industry. This paper discusses the construction technology and management of power engineering, and hopes to provide assistance for the development of China's power engineering.
\end{abstract}

\section{Introduction}

With the acceleration of economic construction, the development of China's power industry has achieved remarkable results. In the power field, construction technology and construction management work play a decisive role in the smooth development and good operation of power engineering. Among them, construction technology is the foundation of power engineering development, and construction management is the guarantee to improve the efficiency of power engineering operation. Actively researching advanced construction techniques and continuously strengthening engineering management are issues that power engineering units have been exploring. At present, with the deepening of China's power reform, the technical and management work of power engineering has also exposed certain deficiencies. For example, the technical work is not perfect, the temporary project management is insufficient, the management process is not systematic, and the power engineering unit must strengthen reform. Strength, improve technical standards, and promote the development of power engineering.

\section{China's Power Engineering Technology Standards}

In the construction of electric power engineering, technical disclosure is an important way to ensure construction technology, scale, engineering characteristics and construction significance; in the process of technical disclosure, it is necessary to clarify the construction process, tasks, quality standards, operation methods, civilized and safe construction requirements of electric power engineering, Relevant measures such as means of saving and quality assurance, while implementing the quality standards for power engineering construction, promote project quality standards. At the same time, according to the scope of application of power engineering construction, the corresponding power engineering technology will be delivered; according to the requirements of engineering technology for the "maintenance management" of power engineering, the project manager and related construction unit groups and technicians must conduct inspection and supervision techniques. The corresponding technology is at the end. In this process, the construction unit is mainly carried out by the individual enterprises of each contracted project. Each technical person in charge ensures the smooth implementation of the construction technology through the strict organization of technical disclosure; in special cases, the technical delivery is completed through the request of the higher level. The technical personnel at different levels shall supervise and review the work under the supervision through construction management to ensure the smooth progress of technology delivery. 
In addition, the management regulations are an important part of the technical disclosure, and the technical disclosure is the core link of the construction process. As the main method of power engineering process control, the construction personnel must firmly implement the technical disclosure work while mastering the process. In the preparation stage of power engineering construction, the project managers, unit chief engineers, engineers, site directors and construction technicians of different departments have passed the strict technical disclosure work to grasp the specific contents of the project; through the organization to participate in the cross-border work, if necessary, carry out different work Organize and revise; to ensure that the technology has been smoothly carried out by promptly informing the relevant approver and the auditing department of the approved plan. After the completion of the technical disclosure, the power engineering personnel will ensure that the power project is under construction by signing relevant key records. When the signature is not approved by the person, the content of the content cannot be changed. When the technical disclosure is needed, it must be changed. Signature, the content of the change must also be approved by the relevant personnel; through different departments, departmental technical personnel, technical personnel, technical inspection and supervision, the phenomenon of not complying with the construction technology, must immediately discourage, when dissuasion can not get a unified result At the time, it should be reported to the higher authorities immediately to stop the construction; when the wrong ground causes equipment, personal safety and quality accidents, the person in charge must be fully responsible. Secondly, when some construction workers violate the standards of the meeting, the construction personnel and the person in charge of construction are directly responsible for the relevant responsibilities. Before the commencement of power engineering, the construction unit and the project manager department must strictly follow the technical work, and strictly follow the equipment specifications, design documents, construction techniques and quality plans.

\section{Current Status of Power Engineering Construction Technology and Management}

At present, in the construction of electric power engineering, the construction technology is still not in place, and the construction technology is the premise and foundation of the smooth construction of the project. The construction technology exists in the construction of the entire electric power project. If the construction technology level is not high, the construction of electric power engineering Quality is also difficult to guarantee. If there is a problem in a certain part of the construction process, the entire power engineering construction will be affected [1]. The technical work can provide guarantees for the construction technology, scale and construction of the project, which enables the construction personnel to better understand the actual situation of the project construction, have a more scientific grasp of the project construction, and adopt more scientific and rational technology in the construction to promote The smooth realization of the goal of power engineering construction, technical work is an indispensable part of the construction of the project, but in the actual construction of power engineering, the technical work is not in place, and the construction of power engineering can not achieve the expected results. At the same time, there are certain problems in the power engineering management work. The power engineering management mainly manages and controls the power engineering construction contracts, personnel and cost, so that the power engineering can be successfully completed. However, in the actual management work, the problems are still emerging. During the signing of the construction contract, many of the auxiliary equipment descriptions for electric power construction were not in place. As a result, equipment procurement was required during the construction process, which adversely affected the progress and cost of the construction. In addition, the construction personnel are not in place at the time of selection. The construction personnel are the main body of the project construction. Their quality ability will directly affect the effect of the electric power construction. The construction personnel's choice is relatively random, and the construction personnel's construction supervision and management is not in place. Can not play the real role of the construction workers. 


\section{Countermeasures of Power Engineering Construction Technology and Management}

Power engineering construction technology management is an important guarantee for power engineering construction, and can play a certain role in the construction of electric power engineering. Therefore, before the construction of electric power engineering, the design unit needs to carry out technical disclosure to the construction unit, and the engineering design ideas The construction unit is informed in detail and clearly, and the technical requirements, quality assurance measures, etc. [2]. The detailed disclosure can accurately describe every link of power engineering construction, ensuring that each construction link is closely linked to ensure the quality and effect of power engineering construction. At the same time, the construction unit needs to re-examine the design and construction drawings, present the existing doubts and technical problems to the design unit and the owner, prepare the scientific construction plan and submit it to the supervisor and the owner for review, so as to ensure the feasibility of the construction plan and minimize the feasibility. Problems in the construction process. The technical department of the construction unit shall conduct a scientific assessment of the post-audit construction plan, and after clearing the relevant issues, the construction unit shall provide technical disclosure to the construction personnel. In the management of power engineering construction technology, there should be perfect rules and regulations to provide a scientific basis for engineering technology management. The construction personnel's technology is excellent again. If there is no high professional technology and professional ethics, the construction project construction cannot be promoted smoothly. Therefore, the construction workers' sense of responsibility should be strengthened, so that every construction technician can clearly define his or her duties and improve. Responsibility mechanism. Improve the rules and regulations, pay attention to improve the brand awareness of construction workers, pay attention to the quality of engineering construction, and pay attention to the image building of enterprises. The construction personnel should clearly define their own scope of work and tasks, and be able to complete their own tasks in a timely and high-quality manner [3]. While ensuring the construction progress of the project, professional management equipment is used to achieve the management effect.

In the construction of electric power engineering, high-quality technology and management personnel are required to provide scientific protection for electric power engineering construction. The technical and management personnel's quality ability will directly affect the effect of electric power construction and construction, and efforts should be made to upgrade technical management personnel. Ability. Improve the training and evaluation mechanism, so that technical management can establish awareness of improving their own quality, regularly organize technical management personnel to conduct training, and update the knowledge and business capabilities of technical management personnel through technical exchange meetings. At the same time, it is also possible to carry out diversified practical activities so that all personnel can actively participate in it, give full play to its own advantages and promote the smooth progress of electric power construction and construction.

The quality of power engineering construction is the main goal. Quality is an important criterion for measuring the success or failure of a project. Therefore, quality management should be strengthened in the construction of power engineering. Do a good job in technical disclosure, so that construction personnel and management personnel can fully understand the standards and technical solutions for construction and construction, and can achieve standardized construction. For projects that have not completed the technical delivery, construction cannot be carried out. At the same time, strengthen the quality supervision and management of power engineering construction, require professional supervisory and management personnel to inspect the part of the project that has been completed to determine whether it is in conformity with the construction drawings and construction standards, and if it is found that the violations are to be immediately suspended for rectification.

At present, science and technology develop rapidly, and the management of power engineering construction technology should give full play to the positive role of modern technology and equipment, realize information management, improve the precision of engineering design and construction, and enable the smooth construction of power engineering. Use computer software to 
promote the improvement of management efficiency, make management work more standardized, organically combine schedule, resources and cost control, and promote scientific improvement of management.

Optimizing the technical management environment of power engineering and standardizing the quality management of power engineering quality technology is the key to ensuring the sound development of power engineering technology management. Before the power engineering is officially started, the power engineering unit and the design unit should do a good job in designing the bottom of the work. It is necessary to understand the design ideas, construction techniques and quality assurance methods, and clarify the construction technical standards of the power engineering. Then, the construction unit of the electric power project should carry out effective re-measurement of drawings, communicate well with the owners, and exchange relevant technical issues. Only after the owner has reviewed the construction plan can the formal construction begin. In addition, the technical department of the electric power engineering should strengthen the evaluation and review of the construction plan and provide guidance to the technical personnel on site. Finally, power engineering should pay more attention to engineering changes, and further review the design of the project after the change, and impose penalties on privately-changed engineering changes [3].

\section{Conclusion}

With the modernization and the development of science and technology, social groups have put forward higher requirements for power engineering while the spiritual culture and material culture are growing. Therefore, in the construction management of power engineering in the new era, it is necessary to continuously strengthen the construction technology and management system; to promote the development of power engineering from various aspects in view of existing problems and construction requirements, and improve the efficiency of internal operation and management of enterprises.

\section{References}

[1] Cao Daming.Some Thoughts on Strengthening Construction Technology and Management of Electric Power Engineering [J].Science \& Technology and Enterprises, 2012(23).

[2] Zhong Yuemei. Thoughts on Technical Management in Power Engineering Construction [J]. Science and Technology Rich Guide, 2012 (9): 119.

[3] Yao Qianli. Power Engineering Construction Technology and Management [J]. Corporate Herald, 2010 (4): 290.

[4] Jiang Zongji. On the construction technology and management of electric power engineering [J]. Science and Technology Innovation Guide, 2009 (14): 75-75.

[5] Feng Jie. Construction Technology and Management of Electric Power Engineering [J]. Urban Construction Theory Research (Electronic Edition), 2012 (20). 